
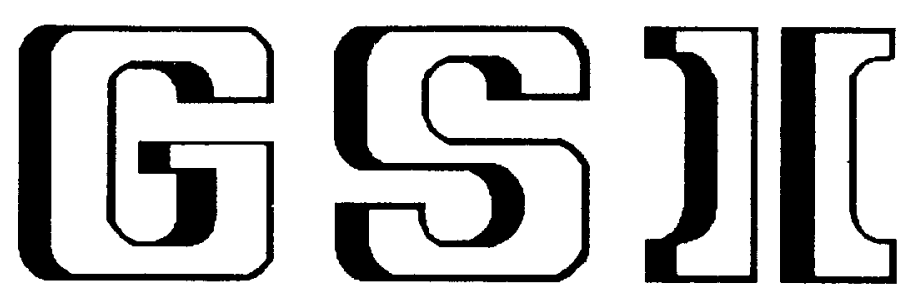

\title{
RECENT DEVELOPMENTS
}

AT THE GSI ON-LINE MASS SEPARATOR

K. Burkard, R. Collatz, M. Hellström, Z. Hu, W. Hüller, O. Klepper,

R. Kirchner, E. Roeckl, K. Schmidt, M. Shibata, A. Weber

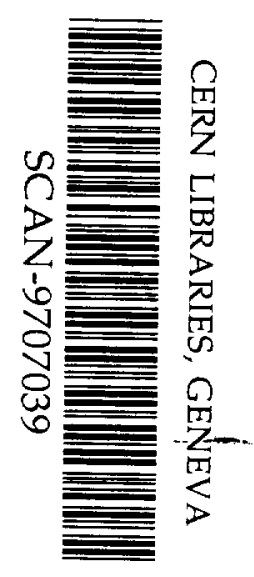

\section{COUPLING A TOTAL ABSORPTION SPECTROMETER}

TO THE GSI ON-LINE MASS SEPARATOR

M. Karny, J.M. Nitschke, L.F. Archambault, K. Burkard, D. Cano-Ott,

M. Hellström, W. Hüller, R. Kirchner, S. Lewandowski, E. Roeckl, A. Sulik

RELEASE STUDIES OF ELEMENTARY AND METAL-FLUORIDE IONS AT THE GSI ON-LINE MASS SEPARATOR

R. Kirchner

Contributions to 13th int. Conf. on Electromagnetic isotope Separators and

Techniques Related to their Applications (EMIS-13), Bad Dürkheim, 22-27 September 1996.

(to be published in Nuclear Instruments and Methods in Physics Research, Section B)

Gesellschaft für Schwerionenforschung $\mathrm{mbH}$

Planckstraße 1 - D-64291 Darmstadt • Germany

Postfach 110552 - D-64220 Darmstadt • Germany 


\title{
Coupling a total absorption spectrometer to the GSI on-line mass separator
}

\author{
M. Karny ${ }^{a}$, J.M. Nitschke ${ }^{b}$, L.F. Archambault ${ }^{b}$, K. Burkard ${ }^{c}$. \\ D. Cano-Ott ${ }^{d}$, M. Hellström ${ }^{c, 1,2}$, W. Hüller ${ }^{c}$, R. Kirchner ${ }^{c}$, \\ S. Lewandowski ${ }^{a}$, E. Roeck $l^{c}$ and A. Sulik ${ }^{a}$ \\ a Institute of Experimental Physics, University of Warsaw, Poland \\ b Lawrence Berkeley National Laboratory, Berkeley, USA \\ c Gesellschaft für Schwerionenforschung mbH, Darmstadt, Germany \\ d Instituto de Física Corpuscular IFIC, Valencia, Spain
}

\begin{abstract}
A total absorption spectrometer, constructed and briefly operated at the on-line isotope separator OASIS at Lawrence Berkeley Laboratory, has been moved and installed at the on-line mass separator of GSI Darmstadt. At GSI, the spectrometer has been used for $\beta$ decay strength function measurements of nuclei in the vicinity of ${ }^{100} \mathrm{Sn}$ and ${ }^{146} \mathrm{Gd}$.
\end{abstract}

Key words:

PACS: 07.85.N, 29.30.-h, 29.40.Mc

EMIS-13, NIM-B, proceedings, LATEX,

\section{Introduction}

Although total absorption $\gamma$-ray spectrometry was introduced over 25 years ago, it remains a powerful tool to study decays processes followed by lowintensity $\gamma$-transitions. This disintegration pattern is often encountered for $\beta$-decays far from stability: here the decay encrgy windows are large and highlying states in the daughter nucleus can be fed. In many cases, most of the $\beta$-feeding goes to regions of high level density. The individual $\gamma$-transitions deexciting these levels are generally too weak to be efficiently detected in highresolution experiments using standard $\mathrm{HPGe}$ detector setups. In contrast, high

1 Corresponding author. E-mail M.Hellstroem gsi.de

2 Supported by the Alexander von Humboldt Foundation.

Article published in Elsevier Preprint EMIS-13 Editor's no. XXX, NIMB (1996) 1 
detection efficiency can be obtained with large $\mathrm{NaI}(\mathrm{Tl})$ crystal spectrometers. One of the important applications of total $\gamma$-ray spectroscopy is the determination of 3 -decay strength functions $S_{3}$, especially for Gamow-Teller decays in the vicinity of closed-shell nuclei [1]. The relatively poor energy resolution of $\mathrm{NaI}(\mathrm{Tl})$ (as compared to $\mathrm{Ge}$ ) can then be accepted, since in order to determine the gross structure of $S_{3}(G T)$ it is generally sufficient to measure the $\beta$-feeding distribution $\beta(E)$ over rather wide energy intervals (e.g. $100 \mathrm{keV}$ ).

In the following, the coupling of a large, Z-selective NaI(Tl) total absorption spectrometer to the on-line mass separator at GSI Darmstadt is described. The individual detector components are presented together with the spectrometer's light-pulser based stabilization system. The performance of the spectrometer relative to similar detectors is briefly discussed.

\section{Technical description}

\subsection{TAS set-up at the GSI on-line mass separator}

After the shutdown of the SuperHILAC at the Lawrence Berkeley National Laboratory, a Total Absorption Spectrometer (TAS) [2] constructed and briefly operated at the OASIS on-line separator was shipped to GSI, together with beam line components, collection chamber and transport tape system. In order to accomodate the spectrometer at the GSI on-line mass separator [3], a special air-conditioned hut was constructed above the floor level of the experimental hall. The cooled environment helps minimize the influence of temperature changes in the experimental hall on the spectrometer performance. Fig. 1 shows the spectrometer in its present location, while a schematic view of the setup is presented in Fig. 2. The separator central beam line has been extended to deflect the mass separated beam $90^{\circ}$ upwards by means of an electrostatic mirror. Electrostatic Einzel- and quadrupole lenses allow to focus the beam onto the implantation point inside the collection chamber. Alternatively, the beam can be redirected by means of an electrostatic deflector to a separate measurement position (on the ground floor) equipped with a collection tape. Here, the composition of the secondary beam can be periodically checked using HPGe detectors, usually in parallel with background measurements performed in the TAS, see below.

Radioactive sources are produced by implanting the mass separated beam into a plastic tape. The sources are then transported to the center of TAS (which is in air) via a differential pumping system. The pressure increases from $\leq 10^{-6}$ mbar in the separator beam line to $\leq 10^{-5}$ mbar in the collection chamber, which is then connected to atmospheric pressure via a series of narrow slits 
(1.7 $\mathrm{mm}^{2}$ rectangular cross section, $105 \mathrm{~mm}$ long): $\leq 10^{-2}$ mbar after the first slit, $\leq 10$ mbar after the second, and finally atmospheric pressure.

To minimize the contributions from external radioactivity, the bottom and side surfaces of the main crystal are shielded with layers of lead $(5 \mathrm{~cm})$ and borax $(7.5 \mathrm{~cm})$. The total rate from room background and cosmic rays amounts to 600 counts per second. The contribution from beam-induced radiation (mainly from $(n, \gamma)$ reactions), which shows the same time structure as the pulsed LNIL.AC beam (see Fig. 3), can be suppressed, e.g., by interrupting the measurement during the beam pulses. The background has to be measured periodically. throughout each experiment; this can conveniently be done in parallel to the source composition checks at the complementary HPGe detector station. The beam distribution between the two setups can be varied from 1:1 to 1:10, as defined by the importance of the background measurements.

\subsection{The TAS detectors}

The TAS is shown schematically in Fig. 4. The main component is a large ( $0356 \mathrm{~mm} \times 356 \mathrm{~mm}$ ) $\mathrm{NaI}(\mathrm{Tl})$ crystal for the detection of $\gamma$-rays. Extending to the center of this "main crystal", a cylindrical well $(051 \mathrm{~mm} \times 203 \mathrm{~mm})$ drilled along the cetector's symmetry axis allows the insertion of a separate detector assembly. In addition to guiding rolls for the tape transporting radioactive sources into the center of the TAS, the standard assembly consists of two $(018 \mathrm{~mm} \times 0.5 \mathrm{~mm}$ or $\varnothing 18 \mathrm{~mm} \times 1.0 \mathrm{~mm})$ silicon energy-loss detectors for $\beta$ particles and a small $(016 \mathrm{~mm} \times 10 \mathrm{~mm})$ cryogenically cooled germanium $X$ ray detector, see the insert in Fig. 4. The radioactive sources are positioned in the center between the two Si counters. A $(043 \mathrm{~mm} \times 150 \mathrm{~mm}) \mathrm{NaI}(\mathrm{Tl})$ "plug" crystal, optically decoupled from the main crystal, completes the near $4 \pi$ geometry. Complementary (plastic) absorbers can be introduced to prevent positrons from penetrating into the main crystal.

The volume of the TAS described here is larger by a factor of 10.0, 5.6 and 2.3 than the spectrometers described in Refs. [5], [6], and in [7], respectively. (The effects of crystal volume on detector performance are discussed below.) A severe limitation of the previous instruments was the lack of $\mathrm{Z}$ identification that might lead to the superposition of spectra from different isobars. This is remedied in the present setup by requiring coincidences with the characteristic $\mathrm{X}$-rays following EC decay recorded in the Ge detector, allowing simultaneous and independent study of the EC strength distributions of several isobars in the same experiment.

If the radioactive sample is of high purity, also the $\beta^{+}$component of the decay can be extracted from the $\gamma$-ray spectrum measured in the $\mathrm{NaI}(\mathrm{Tl})$ 
detectors in coincidence with signals from the Si detectors. In addition, the top Si counter together with the Ge detector may serve as an $\Delta \mathrm{E}$ - $\mathrm{E} \beta$-particle telescope. By gating on a strong $\gamma$-line in the $\mathrm{XaI}(\mathrm{Tl})$ spectrum, the $\beta$ energy spectrum associated with that particular level can be obtained and used for determination of the endpoint energy and $Q_{E C}$ value.

The essential electronic components are shown in Fig. 5. The VaI(Tl) light output is collected by 12 active-base photomultiplier tubes (P.ITs): eleven coupled to the bottom of the main crystal and one to the plug detector. Each PXT base is connected to a computer controlled high voltage supply via two inputs; the first defines the operating point and the second allow's fine control of the gain. The desired total absorption spectrum is obtained by summing the twelve anode outputs (in hardware and/or in software). A second anode output of each PXIT is connected to individual ADCs and forms part of the stabilization system described below.

\subsection{TAS gain stabilization}

To maintain the gain matching between the individual PMTs and avoid amplification shifts due to, e.g., temperature and count rate changes, the PMT outputs are continuously monitored and stabilized relative to a light-pulser reference circuit operated at a frequency of $0.5-1 \mathrm{kHz}$. The output from an amplitude- and color-stabilized light pulser (LP) is transferred to the detector via six randomized-fiber bundles: five to the main crystal and one to the plug. A seventh optical cable is connected to a separate $\mathrm{NaI}(\mathrm{Tl})$ crystal that serves as a reference for the light pulser itself. This $(076 \mathrm{~mm} \times 76 \mathrm{~mm})$ "reference detector", which was manufactured from the same NaI(Tl) material as the main TAS crystal to assure similar optical and temperature responses, is read out via an equivalent PMT. The LP amplitude is continuously compared to a $\gamma$-line from a long-lived isotope (i.e., the $2.506 \mathrm{MeV}$ sum peak of ${ }^{60} \mathrm{Co}$ ) placed in the centre of the reference detector, using a feedback system with two parallel digital stabilizers. The light output of the pulser is, in general, chosen to be in the range of 5-13 $\mathrm{MeV}$ equivalent photon energy absorbed in the main NaI(Tl) crystal.

The TAS stabilization procedure is carried out continuously by a dedicated computer (IBM PC 386) which uses custom software and hence does not interfere with the measurements. The output of each P.MT is recorded in separate ADCs strobed by the light pulser. After a suitable number of light pulses have been recorded (typically $10^{4}$ ), the LP peak centroids are evaluated by the computer and compared to stored reference values. Correction voltages are calculated and superimposed on the high voltages of the individual PMTs to increase or decrease their gain as required. One correction cycle for all 
PMITs takes about $60 \mathrm{~s}$. During operation the gain-control voltages of all PMTs are displayed graphically as functions of time on the PC screen. This useful feature allows the P.IT performance and stability to be easily monitored, e.g., to ensure that the TAS is not overloaded by excessive count rates. (The maximum count rate is $(2-4) \times 10^{4} \mathrm{~s}^{-1}$, depending on the average $\gamma$-ray energy.)

\section{TAS performance}

For an ideal $100 \%$ efficient total absorption spectrometer, the $\beta$-feeding distribution $3(E)$ is proportional to the $\gamma$-intensity distribution $\gamma(E)$. In a real finite-rolume detector, however, the peak detection efficiency is $<100 \%$, and consequently the response function of the detector becomes dependent on details of the deexcitation pattern of the nucleus for a given excitation energy. Thus, a measured $\gamma$ distribution can only be uniquely unfolded into $\beta(E)$ if the complete level scheme of the daughter nucleus is known. Since this is in general not the case, several approximations, mainly based on statistical model assumptions, have been used in the past $[4,5]$.

The large volume of the TAS $\mathrm{NaI}(T \mathrm{~T})$ crystal $(\approx 35$ liter!) results in a total detection efficiency of $\geq 89 \%$ and a photopeak efficiency of $\geq 55 \%$ for monoenergetic photons between 0.2 and $5.0 \mathrm{MeV}$. (For the energy interval from 0.3 to $1.5 \mathrm{MeV}$, the values are $\geq 95 \%$ and $\geq 66 \%$, respectively.) As a consequence, the total detection efficiency approaches $100 \%$ for high multiplicity events while, unfortunately, the photopeak efficiency diminishes rapidly. Therefore knowledge of the multiplicity is essential for the successful unfolding the total absorption spectra. Fig. 6 clearly shows the effect of the large efficiency: here the X-ray gated energy spectrum of the ${ }^{156} \mathrm{Tm} \beta$-decay as measured with the TAS is compared to data obtained by Gadea et al. [8] with a smaller total absorption spectrometer. It is evident that, in contrast to the previous measurement, already the uncorrected spectrum obtained with a larger detector clearly resembles the shape of the underlying feeding distribution curve.

Another prerequisite for the data evaluation is detailed knowledge about the response function of the spectrometer to (monoenergetic) $\gamma$-rays over the full energy range. In order to map out this response function, measurements of "simple" calibration sources like ${ }^{137} \mathrm{Cs},{ }^{24} \mathrm{Na},{ }^{46} \mathrm{Sc},{ }^{60} \mathrm{Co}$, and ${ }^{88} \mathrm{Y}$ have been carried out. For energy ranges not covered in this way, the response function has to be simulated. Since a reliable simulation of the detector response over the full energy range is essential for the deconvolution procedure that transforms the experimental TAS spectrum into the corresponding $\beta$-decay intensity distribution, the performance of three simulation codes are currently being compared: EGS4 [9], GEANT [10], and Sigma [11]. 


\section{Summary and Outlook}

A total absorption spectrometer originally designed, constructed and briefly operated at LBL, has been coupled to the GSI on-line mass separator. To our knowledge, this TAS outperforms previously used instruments since it combines the high detection efficiency of the very large $\mathrm{NaI}(\mathrm{Tl})$ crystal with a high-resolution Ge X-ray detector as well as Si positron detectors. The measurements performed so far indicate that total absorption spectrometry is still a powerful method for 3 -strength determination which cannot be easily replaced even by modern $\mathrm{HPGe}$ detector arrays. On the other hand, the deconvolution of TAS data requires detailed knowledge on the deexcitation patterns of the decays studied, and thus both techniques, i.e., high-resolution and total absorption spectroscopy remain complementary.

Lp to now, several experiments have been performed with an emphasis on $\beta$-strength studies of nuclei in the vicinity of ${ }^{100} \mathrm{Sn}\left({ }^{103-107} \mathrm{In}\right)$ and ${ }^{146} \mathrm{Gd}$ $\left({ }^{148} \mathrm{~Tb}^{2-}, 9^{+},{ }^{150} \mathrm{Ho}^{2-}, 9^{+}\right.$and $\left.{ }^{156} \mathrm{Tm}\right)$. Naking use of the high $\mathrm{X}$-ray- $\gamma$-ray coincidence efficiency of the TAS, the spectrometer has also been used successfully in an off-line pilot experiment aimed at measuring bremsstrahlung emitted in the FC decay of ${ }^{137} \mathrm{La}$. In the near future the 3 -strength studies around ${ }^{100} \mathrm{Sn}$ and ${ }^{1.46} \mathrm{Gd}$ will be continued. Further off-line studies are also planned.

\section{Acknowledgments}

The authors would like to acknowledge valuable contributions from Dr. M.A. Stoyer and P.A. Wilmarth (Berkeley) as well as the participants of the GSI Experiments U83 and U85. This work was supported in part by the European Community under contracts ERBCIPDCT940091 and ERBFMGECT950083, by KBN (Poland) under grant 2-P302-14806, and by CICYT (Spain) under contract AEN94-0833-CQ2-01.

\section{References}

[1] E. Roeck], Proc. Int. Nucl. Phys. Conf., Beijing, China, 1995, Sun Zuxun and Xu Jincheng (eds.) (World Scientific, Singapore, 1996) p. 526.

[2] J.M. Nitschke et al., GSI Annual Report 1994. GSI 95-1 (1995) 285.

[3] C. Bruske et al., Nucl. Instr. Meth. 186 (1981) 61; K. Burkhard et al., contribution to this conference.

[4] C.L. Duke et al., Nucl. Phys. A151 (1970) 609. 
[5] B.R. Erdal and G. Rudstam, Nucl. Instr. Meth. 104 (1972) 263.

[6] G.D. Alkhazoy et al., Phys. Lett. B157, 350 (1985).

[7] R.C. Greenwood et al. Nucl. Instr. Meth. Physs. Res. A314 (1992) 514.

[8] A. Gadea et al. Proc. of Int. Conf. Future of Nuclear Spectroscopy, Crete, Greece, 1993.

[9] W.R. Nelson et al., SLAC Report 265, December 1985.

[10] Developed by the CERN CPC Division.

[11] V. Wittmann et.al., private communication. 


\section{Figures}

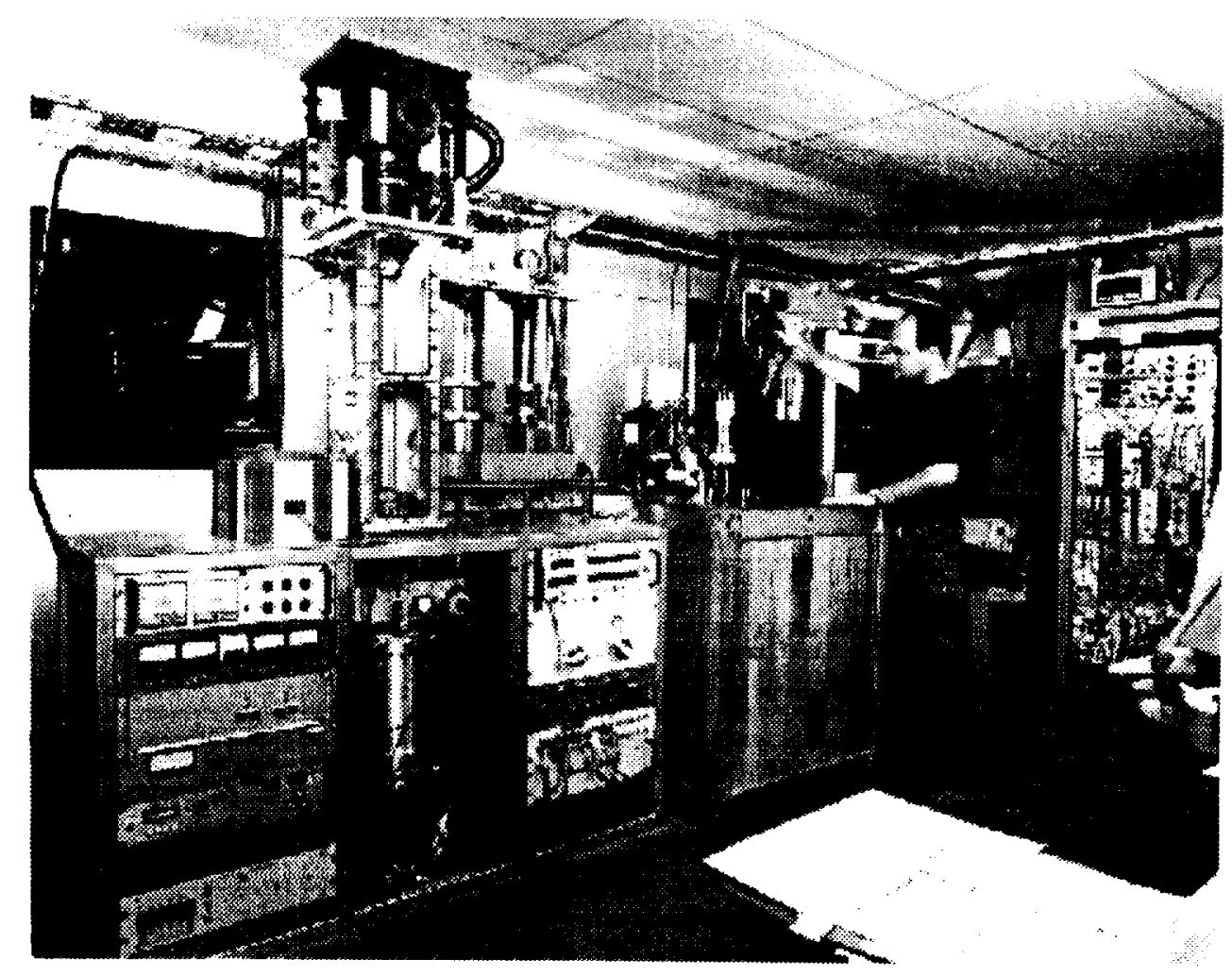

Fig. 1. The TAS in its present location. Note the collection chamber with its differential pumping system on the left, spectrometer (inside its lead and borax shiclding) in the center, and part of the readout electronics to the right. (Photo: $\mathrm{A}$. Zschau, (GSI) 


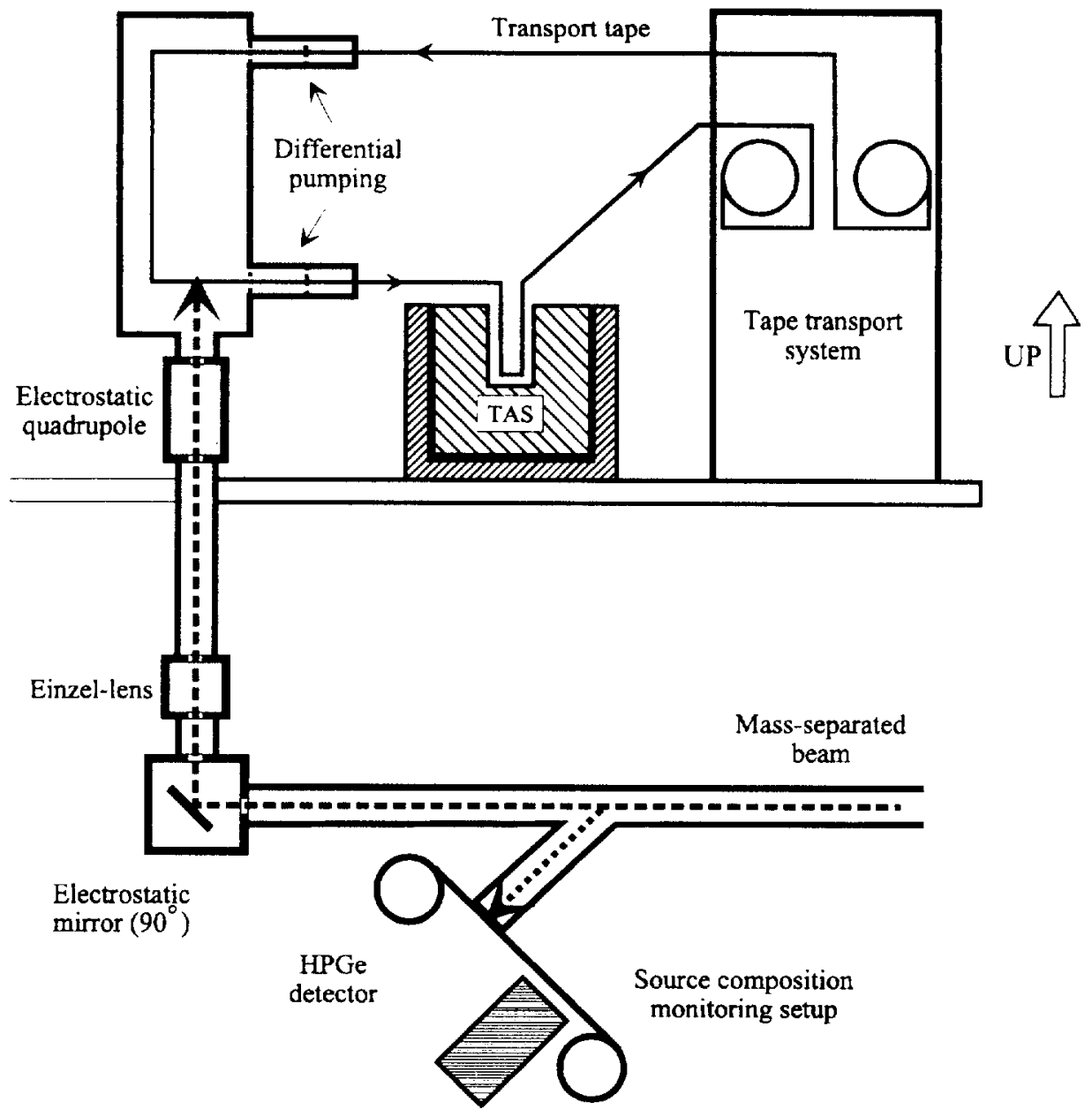

Fig. 2. The coupling of the TAS to the GSI on-line mass separator. The mass separated beam can either be directed up to the TAS platform or to the monitoring setup on the ground floor. 


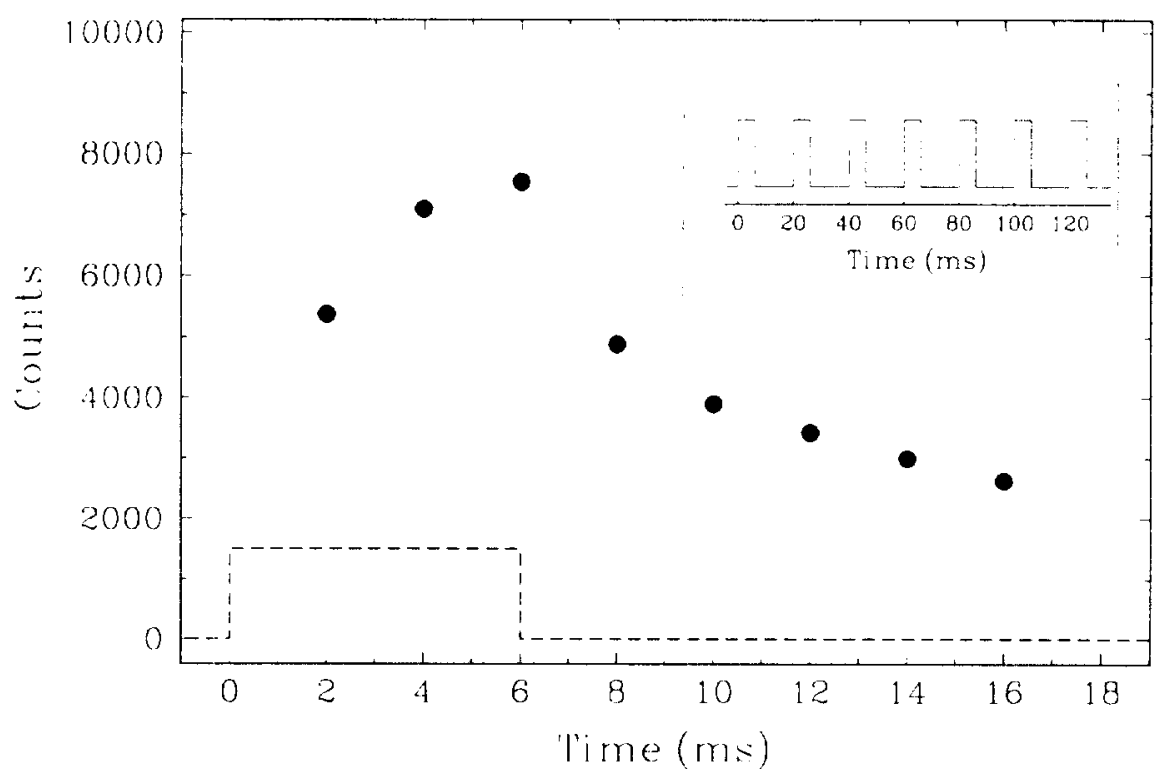

Fig. 3. The time dependence of the background rate in the TAS main Nal(Tl) crystal measured with a $10.2 \mathrm{MeV} /$ nucleon, $6 \times 10^{12}$ ions $/ \mathrm{s}^{12} \mathrm{C}$ beam impinging on the mass separator target. The dashed line indicates the duration of one beam pulse. The insert shows the macrostructure of the UNILAC beam. 


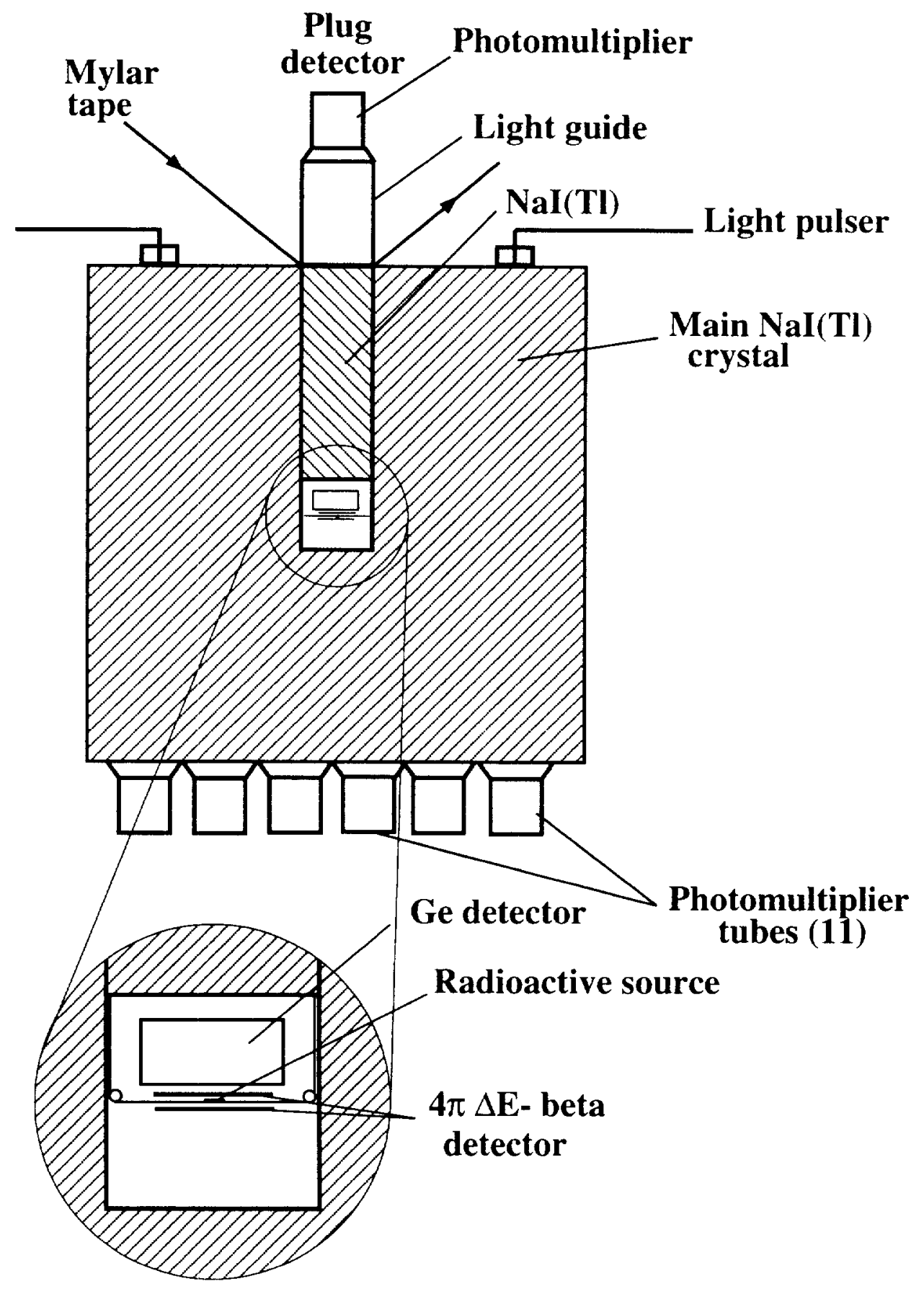

Fig. 4. A schematic cross section view through the center of the TAS. The insert shows the Si and Ge detectors surrounding the source position. 


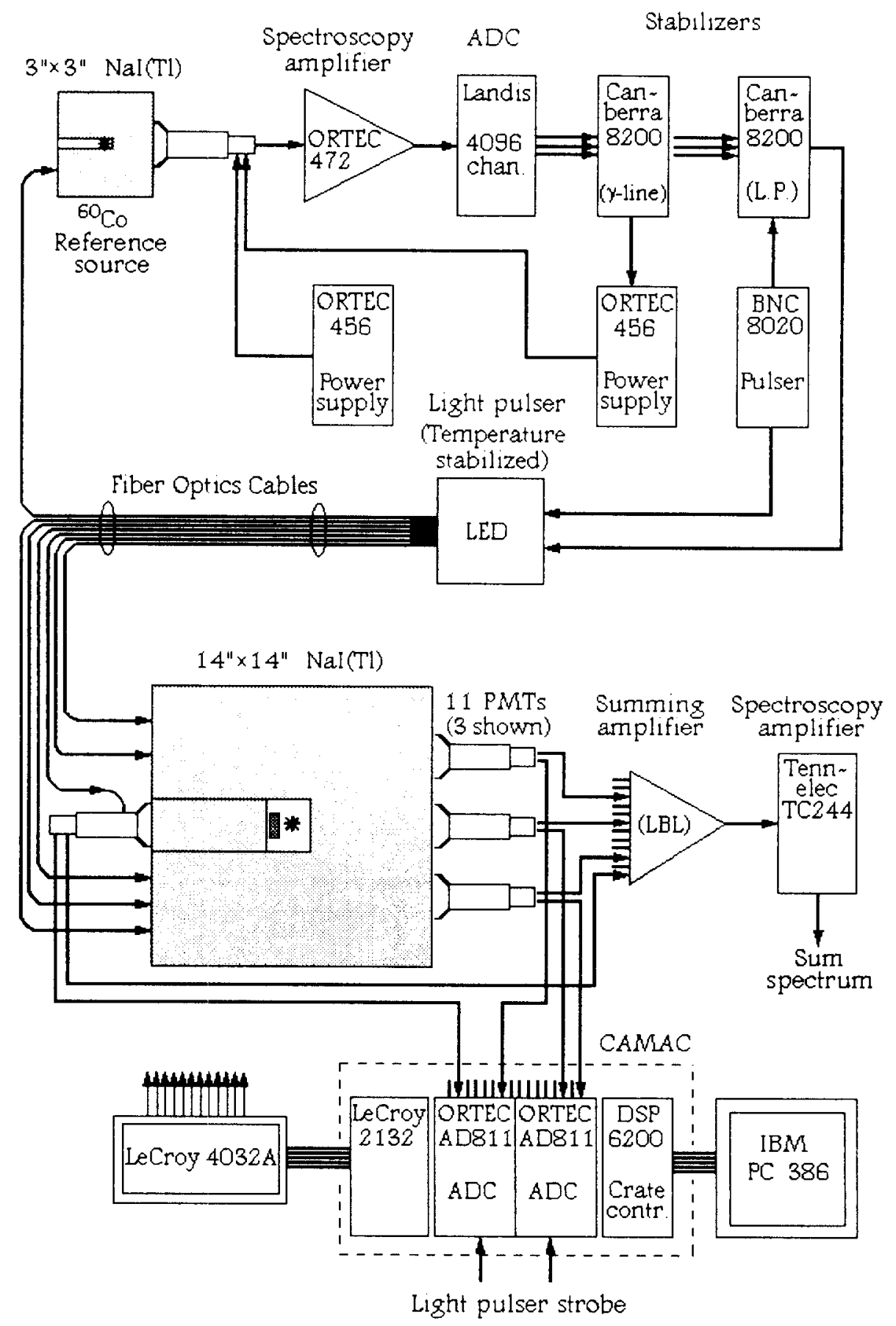

Fig. 5. Schematic of the TAS electronics, including the light pulser stabilization circuit. 


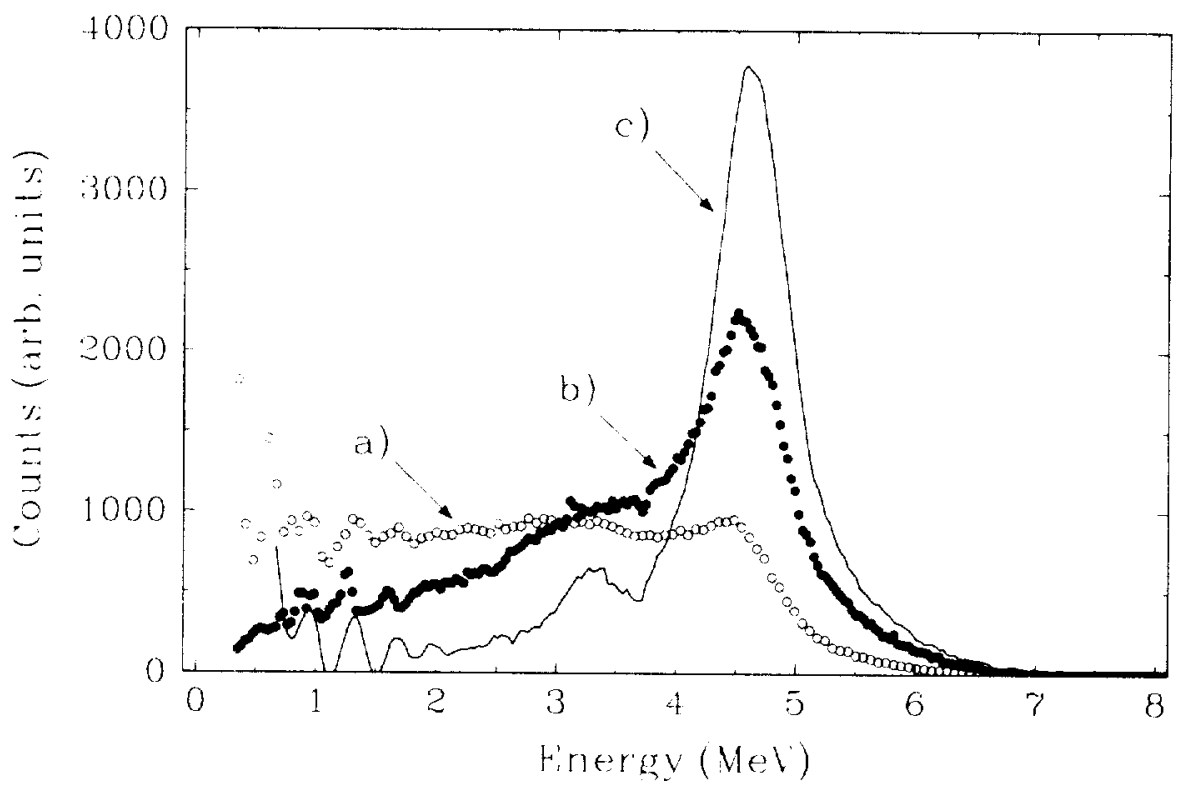

Fig. 6. ${ }^{156} \mathrm{Tm}$ EC decay: A comparison between the "raw" $\gamma$-ray spectra measured with (a) a $(0203 \mathrm{~mm} \times 203 \mathrm{~mm}) \mathrm{NaI}(\mathrm{Tl})$ crystal[8] and (b) the $(0356 \mathrm{~mm} \times 356 \mathrm{~mm})$ $\mathrm{NaI}(\mathrm{Tl})$ detector of the TAS. Curve (c) shows the EC feeding distribution deduced from spectrum (a). 
